posed of morbid structures in conjunction with isolated portions of perfectly-formed animal organs, having no other relation to the living being with which they are connected, than as they are dependent upon it for the means of nutrition and growth; these cases, it is added, must be considered to belong to the class of parasitic monsters.

Thirdly, the cases wherein the tumor being of the nature of spina bifida, consists of a membranous cyst, communicating with the theca vertebralis.

Fourthly, the cases wherein the tumor consists wholly or in part of membranous cysts communicating with the spinal canal, but externally to the theca, between this and the surrounding bony walls of the canal.

In all these cases here described, and in others to which reference is made, the tumor was attached to the external and posterior part of the walls of the pelvis, and consequently its situation was guch as to allow of its removal by operation, provided there was no objection either from the depth of its attachments, or from the continuity of any part of it with the membranes of the spinal marrow, or other of the external organs of the body of the child to which it was united.

In one of the cases here related, the congenital tumor of large size was removed with complete success, by the late Mr. Thomas Blizard. The tumor, which is preserved in the Museum of the Royal College of Surgeons, consists of distinct solid fibrous substances, and of an isolated portion of intestine three and a half inches in length, closed at each end, and having at one end a narrow process continued from it, of the exact form of an appendix vermiformis. In the process of the removal of the tumor, this intestine was opened, and there flowed from its interior a fluid closely resembling meconium in its colour and consistence. The author of the paper states, that an analogous fact of the production of a fluid exactly like meconium in its appearance, without the existence of a liver, or other distinct hepatic apparatus, occurred to his observation in the dissection of an acephalous lamb, in which, with other malformations, the liver was wanting, and the intestines were filled by a thick fluid, darkcoloured, but which, when diluted, presented the yellow colour of bile, but it had not a bitter flavour.

READ Also, PATHOLOGICAL AND SURGICAL OBSERVATIONB ON THE DIBEASES OF THE EAR, BY JOSEPH TOYNBEE, RSQ., PRESENTED BY DR. R. BRIGHT.

The present paper is the first of a series which the author hopes to lay before the Society on the same subject, and contains the details of forty-one dissections of the internal ear in patients who have died in hospitals and infirmaries of various diseases, and of whose faculty of hearing, as to the greater number at least, the author was uninformed. The following is a concise view of the state of the cavity of the tympanum in these cases :-

1. In a healthy state . . . . . . . . 10

2. With simple thickening of the investing

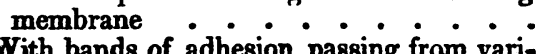

3. With bands of adhesion passing from various parts of the cavity of the tympanum most frequently connecting the stapes to

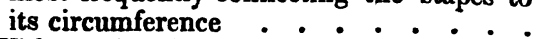

4. With slight thickening of the investing membrane, accompanied by the existence of adhesive bands . . * . . . . 13

5. With considerable thickening of the investing membrane, and bands of adhesion . 5

6. With suppuration of the cavity of the tympanum . • • • . . . . . . 1

7. With anchylosis of the base of the stapes to the circumference of the fenestra ovalis 2

"The large proportion of specimens which are undoubtedly in a diseased state," says the author, " is very surprising, but it may be less so, when I state that many persons whom I have examined, and who have considered that they hear perfectly well, cannot distinguish the ticking of my watch at the distance of two and a half feet, and in some cases at four or five inches only, though the same watch can be heard by a healthy ear seven or eight feet from the head. I am therefore disposed to believe that the function of the ear is impaired much more frequently than is generally supposed."

The author concludes his paper, by inviting the members of the profession to inspect the preparations described in his paper. In consequence of there being still many papers unread, there will be an extra meeting of the Society on 'Tuesday next, the 6 th inst.

\section{ACADEMY OF SCIENCES.}

Paris, June 14.

POIBONING WITH ARSENIC.

The various questions relative to poisoning with arsenic and Marsh's apparatus, which originated at the trial of Madame Laffarge, were submitted to a committee of the Academy of Sciences; the following is the report of the committee.

1. Marsh's apparatus is capable of easily detecting the rowowth part of arsenious acid in a fluid; the spots begin to appear when the fluid contains only 2000000.

2. The spots are not more readily produced with a large quantity of fluid than with a small one, if each contain a relative proportion of arsenic; but in the former case, they', are deposited during a longer time. Hence it is advantageous to operate on a small quantity of fluid, as the spots will be much more intense.

3. It is a matter of the utmost importance to make the gas pass through a tube at least eleven inches long, containing cotton, for the purpose of arresting the matter which is always carried along mechanically by the gas: unless this be done, we run a risk of obtaining spots of the oxysulphuret of zinc, which often resemble those of arsenic.

4. The process proposed by $M$. Lassaigne may be useful. It consists in passing the arsenuretted hydrogen through a neutral solution of the nitrate of silver, and then decomposing by hydrochloric acid; the acids are eliminated by evaporation, and the residuum tested for arsenic. This method is particularly convenient for operating on small 
quantities of fluid ; we must, however, be on our guard against concluding the presence of arsenic, simply because the solution of nitrate of silver becomes troubled, or throws down a precipitate during the passage of the gas through it ; for this may occur from other gases than the arsenious, or even from hydrogen itself, under the influence of light. The solution of nitrate of silver may be replaced by a solution of chlorine, or an alkaline chloride.

5. The method pointed out by Berzelius and Liebig, and usefully modified by Kœppelin and Kampmann, is capable of discovering very minute quantities of arsenic that could not be shown in spots; it condenses the arsenic more completely than any other process; however, the arsenic is often mixed with sulphuret of arsenic, which may alter its colour, especially when in very small quantity. It is this method which the committee prefer to all others; the apparatus, they think, should be arranged in the following manner.

The flask must have a straight neck with a large mouth ; the cork is pierced by two holes : through one a straight tube, (of 4.433 lines in diameter) is passed to the bottom of the flask; through the other a smaller tube, bent at right angles. The latter passes into another tube, eleven inches long, filled with asbestos ; this latter joins a tube of very small diameter, (one line,) and pointed at the end; the extremity of this tube, for about three inches, is enveloped in a metallic leaf. The flask must be chosen of sufficient size to contain one-fifth more than the quantity of fluid operated on; and when the latter is supposed to contain only traces of arsenic, that quantity should be small. The extremity of the tube in the flask is cut off obliquely, and furnished with a small bulb at any point of the vertical branch, for the purpose of condensing any water which may be carried up. The apparatus being thus arranged, a few bits of zinc are introduced into the flask, and then some water, sufficient to cover the end of the safety tube; then some sulphuric acid is poured in ; the air contained in the flask is expelled by the hydrogen. The end of the horizontal tube is now heated red with charcoal, and the suspected fluid introduced into the flask with a funnel, care being taken that no air enters with it. Should the gas be disengaged more slowly after the introduction of the fluid, some more sulphuric acid must be added, and the process must be carried on in as regular a manner as possible. If the gas contain any arsenic, the latter will be deposited in the form of a ring, around the extremity of the heated tube; and the gas which escapes may be ignited, so as to collect any spots on a porcelain cup; for a portion of the metal may be carried over, unless the tube be heated for a sufficient length. The tube might be bent, and the end passed into a solution of the nitrate of silver, so as to collect every particle of arsenic.

As the metal is deposited in the form of a ring round the tube, we can easily examine all its physical and chemical properties.

1. Its volatility. 2 . Its conversion by heat into arsenious acid. 3. By heating a little nitric acid in the tube, the arsenic is converted into arsenious acid, soluble in water. On carefully evaporating the water in a small porcelain cup, we obtain a brick-red precipitate with a neutral solution of nitrate of silver. 4. Having applied all these tests, we may again obtain metallic arsenic. A small quantity of black flux is placed in the capsule containing the brickred residuum; the contents are dried and introduced into a small tube, one end of which is pointed, the other closed. On heating the tube red, the arsenic is converted into a metallic state, and forms a ring round the narrow end of the tube.

6. The zinc and sulphuric acid obtained in shop, may be frequently employed without furnishing traces of arsenic in Marsh's apparatus. The sulphuric acid employed by the committee was purified by distillation, and the zinc by being reduced to fine scales. In all cases, the analyst should test the purity of the substances which he is about to employ, and after he has tested the fluids or matter supposed to contain poison, he should repeat the same operations, on the same quantities, with his tests, on fluids known not to contain any poison. Thus, if he has carbonised animal matter by sulphuric or nitric acid, he should evaporate in the same kind of vessels similar quantities of these acids, \&c.

7. The process of carbonising animal matter by nitric acid or the nitrate of potass may succeed completely; but sometimes there unavoidably occurs, towards the end of the experiment, a deflagration by which a large part of the arsenic is lost. Carbonising by sulphuric acid, and then treating the carbon obtained with nitric acid, seems to the committee much preferable in a great many cases; this method requires the use of a smaller quantity of the reagent, and does not give rise to so great a waste of arsenic as the others.

8. It is a matter of the utmost importance that the whole of the animal matter should be completely carbonised; unless this be done, we obtain a fluid which froths, and gives spots somewhat similar to those of arsenic ; these were first noticed by $M$. Orfila, under the name of dirt-spots, and are sometimes produced in great quantity, but they can be readily distinguished by proper tests from the true arsenical spots.

9. As to the arsenic which has been said to exist naturally in the human body, all the experiments made by the committee on the bones and soft parts, gave negative results.

10. The committee are of opinion, that when Marsh's apparatus is employed in a proper manner, it is suited to all cases in which the quantity of arsenic is much greater than what the sensibility of the apparatus is capable of exhibiting; it should only be employed with the object of concentrating the metal, and we should reject, or at least regard as very doubtful, any indications furnished by it, when the crust round the end of the tube is too thin to allow the experimenter to apply the chemical tests of arsenic.

In the majority of cases of poisoning with arsenic, the medical jurist will be able to obtain the metal from the matter which has been vomited, or from what remains in the intestines, and will not find it necessary to carbonise the flesh, unles: the former means fail, or in very rare cases. 